\title{
The Mean Values of Totally Real Algebraic Integers
}

\author{
By C. J. Smyth
}

\begin{abstract}
Let $M_{p}(\alpha)$ be the $p$ th root of the mean absolute values of the $p$ th powers of a totally real algebraic integer $\alpha$. For each fixed $p>0$ we study the set $\mathfrak{M}_{p}$ of such $M_{p}(\alpha)$. We show that its structure is as follows: on the nonnegative real line it consists of some isolated points, followed by a small interval in which its structure is as yet undetermined. Beyond this small interval, it is everywhere dense.
\end{abstract}

0. Introduction. Let $\alpha$ be a totally real algebraic integer of degree $d$, with conjugates $\alpha=\alpha_{1}, \alpha_{2}, \ldots, \alpha_{d}$, and for $p>0$ put

$$
M_{p}(\alpha)=\left(\frac{1}{d} \sum_{i=1}^{d}\left|\alpha_{i}\right|^{p}\right)^{1 / p} .
$$

Since $M_{p}(\alpha) \geqslant \mid$ Norm $\left.\alpha\right|^{1 / d}$, it follows that $M_{p}(\alpha)>1$ unless $\alpha=0, \pm 1$. Let $\Re_{p}$ be the spectrum in $(1, \infty)$ of $M_{p}(\alpha)$ :

$$
\mathfrak{N}_{p}=\left\{x \in(1, \infty) \mid x=M_{p}(\alpha) \text { for some totally real algebraic integer } \alpha\right\} .
$$

In this paper we study the structure of $\Re_{p}$. Theorem 1 below gives our main results for certain specific values of $p$, while Theorem 2 gives corresponding (but somewhat weaker) results for all $p>0$.

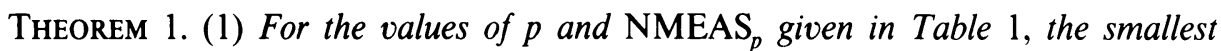
$\mathrm{NMEAS}_{p}$ elements of $\mathfrak{\pi}_{p}$ are isolated, and are the only elements of $\mathfrak{\pi}_{p}$ in $(1$, $\left.\mathrm{MBOUND}_{p}\right)$. These values are the $M_{p}(\alpha)$, where $\alpha$ has minimal polynomial whose number, read from Table 1, corresponds to the polynomial given in Table 2. [For instance, for $p=1, \mathfrak{K}_{1}$ in $(1,1.18119)$ consists of $M_{1}(2 \cos 2 \pi / 5), M_{1}(2 \cos 2 \pi / 7)$, $M_{1}\left(\beta_{2}\right)$ and $M_{1}(2 \cos 2 \pi / 60)$.]

(2) $\mathcal{M}_{p}$ is everywhere dense in the interval $\left(\mathrm{MDENSE}_{p}, \infty\right)$, where

$$
\begin{aligned}
\operatorname{MDENSE}_{p} & =\min \left(a_{p}, c_{p}\right), \\
a_{p} & =\lim _{n \rightarrow \infty} M_{p}\left(\beta_{n}\right), \\
c_{p} & =\lim _{n \rightarrow \infty} M_{p}(2 \cos 2 \pi / n) .
\end{aligned}
$$

Here the $\beta_{n}$ are defined as in [14] by $\beta_{0}=1$ and $\beta_{n}>1$ satisfying

$$
\beta_{n}-\beta_{n}^{-1}=\beta_{n-1} \quad(n \geqslant 1) .
$$

Received November 9, 1982.

1980 Mathematics Subject Classification. Primary 12A95, 12B05. 
(ู)

8
กิ

ह

to

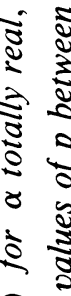

ชิ పे
国

ำำำ

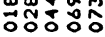

落落

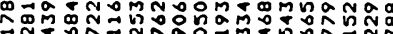

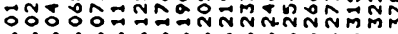

क्ष

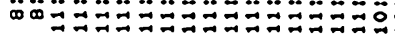

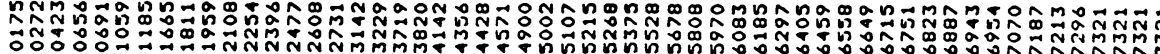

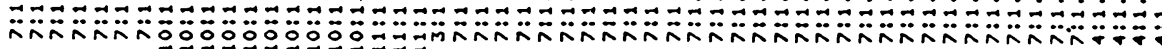

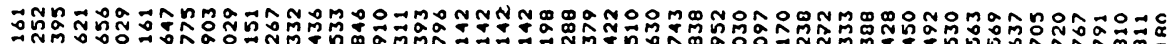

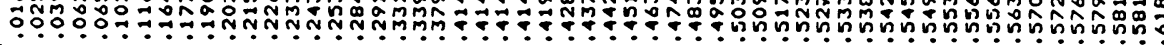

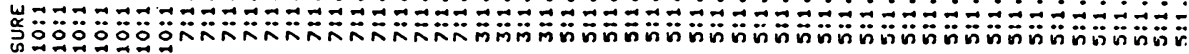

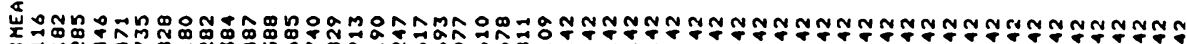

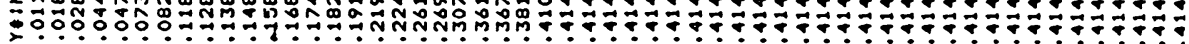

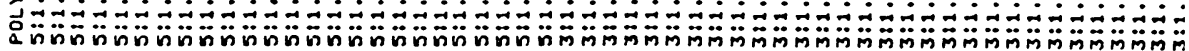
㟧

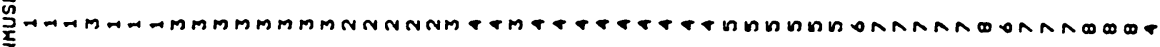
密。

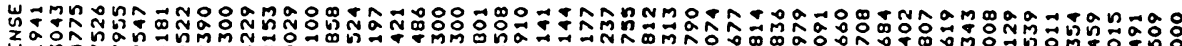

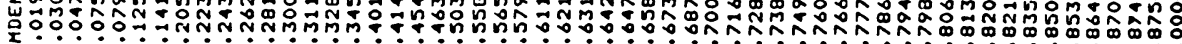

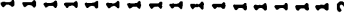

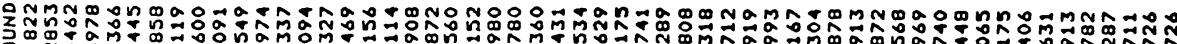

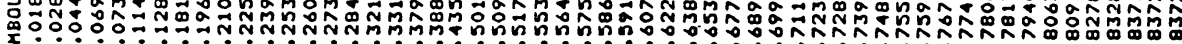

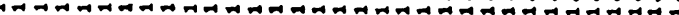

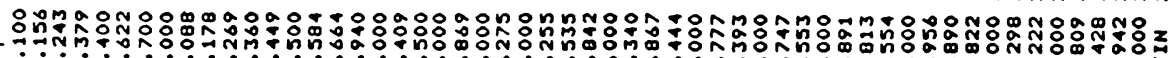

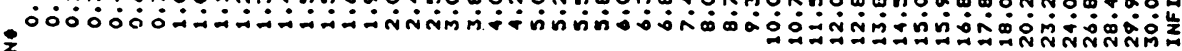
is

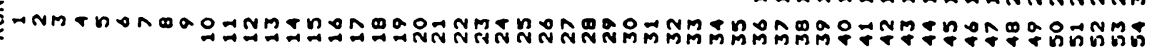


From these results we see that it is only in the interval $\left(\mathrm{MBOUND}_{p}, \mathrm{MDENSE}_{p}\right)$ that the structure of $\Re_{p}$ is undetermined. So the smallest limit point of $\mathscr{N}_{p}$ lies between $\mathrm{MBOUND}_{p}$ and $\mathrm{MDENSE}_{p}$. It is, however, tempting to conjecture that it actually equals MDENSE .

It is also worth noting that $a_{2}=c_{2}=\sqrt{2}$, and, at least numerically,

$$
a_{p}<c_{p} \text { for } 0<p<2 \text {, and } a_{p}>c_{p} \text { for } p>2 \text { (see Section 5). }
$$

For all $p>0$ we have

THEOREM 2. Let $p>0$ be given. Then

(1) If $0<p<0.1, \Re_{p}$ in the interval $(1,1+0.1459 p)$ consists only of the point $M_{p}(2 \cos 2 \pi / 5)$ (see Theorem 5).

(2) Suppose $p \geqslant 0.1$, and let $p^{\prime}$ be the largest value $\leqslant p$ in the $p$-column of Table 1 . Then $\Re_{p}$ in $\left(1, \mathrm{MBOUND}_{p^{\prime}}\right)$ consists of between $\mathrm{NMUSE}_{p^{\prime}}$ and $\mathrm{NMEAS}_{p^{\prime}}$ discrete points, the precise number of points, and to which $\alpha$ they correspond, being calculated with the aid of Table 1. [ For instance, for $p=2.9, p^{\prime}=2.5$ ( Run 20), $\mathrm{NMUSE}_{p^{\prime}}=2$, and there are 2 elements of $\Re_{p}$ in $(1,1.38872)$, namely $M_{p}(2 \cos 2 \pi / 5)$ and $M_{p}(2 \cos 2 \pi / 7)$. However, for $p=2.501, p^{\prime}=2.5$ again, and there are three elements of $\mathcal{M}_{p}$ in $(1,1.38872)$, the third one being $M_{p}(2 \cos 2 \pi / 60)$, this value being less than 1.38872 for $p$ close to 2.5.]

(3) For all $p>0, \mathfrak{M}_{p}$ is dense in $\left(\mathrm{MDENSE}_{p}, \infty\right)$, where $\mathrm{MDENSE}_{p}$ is defined by (0.1).

\section{TABLE 2}

This table shows to which polynomials the POLY \#'s in Table 1 refer. The coefficients given are of the minimal polynomials of $\alpha$, where $\alpha$ has small measure.

\begin{tabular}{ccccrrrrrrrr} 
Poly & $\alpha$ & Degree & \multicolumn{1}{c}{ Coefficients } & & & \\
3 & $\sqrt{2}$ & 2 & 1 & 0 & -2 & & & & & \\
4 & $\sqrt{3}$ & 2 & 1 & 0 & -3 & & & & & \\
5 & $2 \cos 2 \pi / 5\left(=\beta_{1}{ }^{1}\right)$ & 2 & 1 & 1 & -1 & & & & & \\
6 & $2 \cos 2 \pi / 16$ & 4 & 1 & 0 & -4 & 0 & 2 & & & \\
7 & $2 \cos 2 \pi / 7$ & 3 & 1 & 1 & -2 & -1 & & & & \\
8 & $1 /(2 \cos 2 \pi / 7)$ & 3 & 1 & 2 & -1 & -1 & & & & \\
9 & $2 \cos 2 \pi / 9$ & 3 & 1 & 0 & -3 & 1 & & & & \\
10 & $\beta_{2}$ & 4 & 1 & -1 & -3 & 1 & 1 & & & \\
11 & $2 \cos 2 \pi / 60$ & 8 & 1 & 0 & -7 & 0 & 14 & 0 & -8 & 0 & 1 \\
12 & $2 \cos 2 \pi / 11$ & 5 & 1 & 1 & -4 & -3 & 3 & 1 & & & \\
13 & $2 \cos 2 \pi / 13$ & 6 & 1 & 1 & -5 & -4 & 6 & 3 & -1 & & \\
14 & $\beta_{3}$ & 8 & 1 & -1 & -7 & 4 & 13 & -4 & -7 & 1 & 1
\end{tabular}

It is easy to translate the above theorems for totally real algebraic integers into corresponding results for totally positive algebraic integers, using the easily proved fact that for $\alpha$ totally positive

$$
M_{p}(\alpha)=\left(M_{2 p}(\sqrt{\alpha})\right)^{2} .
$$

Previous Results. In 1945 Siegel [13] showed that the smallest point of $\mathfrak{R}_{2}$ is $M_{2}\left(\frac{1}{2}(1+\sqrt{5})\right)=\sqrt{3 / 2}$. Recently McAuley, whose thesis [9] stimulated the present 
paper, found one isolated point of $\mathfrak{S}_{p}$ for $p=1,3$, two isolated points for $p=2$ and all $p \geqslant 4$, and three isolated points for $p=4$ and 6 . The methods used were quite different to those used here. Concerning the smallest limit point $l_{p}$ of $\mathfrak{R}_{p}$, Siegel showed $l_{2} \geqslant 1.3166$, and Hunter [7] showed $l_{4} \geqslant 1.4687$. McAuley improved Hunter's result slightly, also showed that $l_{1} \geqslant 1.1515$, and got inequalities for $l_{3}, l_{6}$, $l_{8}, l_{10}$, and $l_{12}$. He also gave the bound $l_{p} \leqslant \lim _{n \rightarrow \infty} M_{p}(2 \cos 2 \pi / n)$. All these results are superseded by the present paper.

We note that all isolated points of $\Re_{p}(p>0)$ found so far are either of the form $M_{p}\left(\beta_{n}\right)$ or $M_{p}(2 \cos 2 \pi / n)$ for some $n$. It is expected that, for small $p$ (perhaps for all $p<2$ ) there exist other isolated points of $\mathscr{K}_{p}$ : these are the points $M_{p}(\alpha)$ where $\alpha$ is a fixed point of an iterate of $H, H$ being defined by

$$
H x=x-x^{-1} \text {. }
$$

In fact $\alpha=2 \cos 2 \pi / 7$ satisfies $H(H(H(\alpha)))=-\alpha$, and $\alpha=2 \cos 2 \pi / 60$ satisfies $H(H(H(H(\alpha))))=\alpha$. However not all such fixed points are of the form $2 \cos 2 \pi / n$ for some $n$; see [14, p. 148].

The proofs of Theorems 1 and 2 are contained in the following sections. In Section 1 we describe the computation. In Section 2 we show that $\mathscr{M}_{p}$ is dense in $\left(a_{p}, \infty\right)$, and in Section 3 we show that $\mathfrak{M}_{p}$ is dense in $\left(c_{p}, \infty\right)$. In Section 4 we find the smallest element of $\mathfrak{M}_{p}$ for $p<0.1$, a range not covered by the computation. Finally in Section 5 we obtain a recurrence for the limit points $a_{2 k}, 2 k$ an even integer, and show that $a_{p} \rightarrow \infty$ as $p \rightarrow \infty$.

1. The Computation: Theory and Practice. The computational method used here is similar to the one used in [15], where we found the four smallest values of $\Omega(\alpha)=\left(\prod_{i=1}^{d} \max \left(1,\left|\alpha_{i}\right|\right)^{1 / d}\right)$. We make a list of totally positive algebraic integers $\alpha^{\prime}$ with minimal polynomials $P_{1}, P_{2}, \ldots, P_{n}$ say, with $M_{p}\left(\alpha^{\prime}\right)$ small. Then for any totally positive $\alpha$ not on the list, the resultant of $\alpha$ and $\alpha^{\prime}$ is nonzero, so that

$$
\prod_{i=1}^{d}\left|P_{j}\left(\alpha_{i}\right)\right| \geqslant 1 \quad(j=1, \ldots, n) .
$$

Writing $\mu_{\alpha}(x)=d^{-1} \times$ number of $\alpha_{i}$ in $(0, x]$, we can express $(1.1)$ as

$$
\int_{0}^{\infty} \log \left|P_{j}(x)\right| d \mu_{\alpha}(x) \geqslant 0 \quad(j=1, \ldots, n),
$$

and then also

$$
\frac{1}{d} \sum_{i=1}^{d} \alpha_{i}^{p}=\int_{0}^{\infty} x^{p} d \mu_{\alpha}(x) .
$$

Suppose that we can solve for a general probability distribution $\mu$ on $(0, \infty)$ the following optimization problem:

$$
\left\{\begin{array}{l}
\underset{\mu}{\operatorname{Minimize}} y_{p}=\int_{0}^{\infty} x^{p} d \mu(x) \\
\text { subject to } \int_{0}^{\infty} \log \left|P_{j}(x)\right| d \mu(x) \geqslant 0 \quad(j=1, \ldots, n)
\end{array}\right.
$$

and that $m_{p}=\inf _{\mu} y_{p}$. Then $M_{p}(\alpha)^{p}=\sum_{i=1}^{d} \alpha_{i}^{p} \geqslant m_{p}$ for any totally positive $\alpha$ not on the list. 
As in [15] we solve (1.2) by forming the dual

$$
\underset{c_{1}, c_{2}, \ldots, c_{n} \geqslant 0}{\operatorname{Maximize}} \min _{x>0} g(x, \mathbf{c}),
$$

where

$$
g(x, \mathbf{c})=x^{p}-\sum_{j=1}^{n} c_{j} \log \left|P_{j}(x)\right| .
$$

By the simple argument of [15, Section 3], the maximum $T_{p}$ of the dual problem is $\leqslant m_{p}$, so that $M_{p}(\alpha) \geqslant T_{p}^{1 / p}$. The method used to solve the dual will not be discussed here. It is a refined version of that used in [15], and it is intended that it be the subject of another paper. Actually the dual is not quite solved, but a near optimum $T_{p}^{\prime} \leqslant T_{p}$ is obtained, so that still $M_{p}(\alpha) \geqslant T_{p}^{\prime 1 / p}$ (for $\alpha$ totally positive, $\alpha$ not on the list). This result is then translated into a result for totally real $\alpha$ using (0.5) to yield

$$
M_{p}(\alpha)=M_{p / 2}\left(\alpha^{2}\right)^{1 / 2} \geqslant\left(T_{p / 2}^{\prime}\right)^{1 / p}=\operatorname{MBOUND}_{p}
$$

for $\alpha$ totally real, $\alpha^{2}$ not on the list.

\section{TABLE 3}

These polynomials are used as 'resultant constraints' in the computation. Polynomials 3 to 14 are the minimal polynomials of $\alpha^{2}$, for the $\alpha$ in Table 2. The labels e.g. 5c refer to Robinson's list [12], while SALEM $1+2$ refers to

\begin{tabular}{|c|c|c|c|c|c|c|c|c|c|c|c|c|}
\hline$F \cdot O L Y *$ & $F \cdot O L Y+1$ & $M T^{\circ}$ & IIEGP & $\mathrm{Cl}$ & $F F \pm$ & IENTS & & & & & & \\
\hline 1 & $x$ & & 1 & 1 & 0 & & & & & & & \\
\hline 2 & $x-1$ & & 1 & 1 & -1 & & & & & & & \\
\hline 3 & $x-2$ & & 1 & 1 & -2 & & & & & & & \\
\hline 4 & $x-3$ & & 1 & 1 & -3 & & & & & & & \\
\hline 5 & CYC 5 & $* * 2$ & 2 & 1 & -3 & 1 & & & & & & \\
\hline 6 & CYC 16 & $* * 2$ & 2 & 1 & -4 & 2 & & & & & & \\
\hline 7. & CYC & $* * 2$ & 3 & $i$ & -5 & 6 & -1 & & & & & \\
\hline 8 & CYC & $*-2$ & 3 & 1 & -6 & 5 & -1 & & & & & \\
\hline 9 & CYC 9 & $* * 2$ & 3 & 1 & -6 & 9 & -1 & & & & & \\
\hline 10 & HETA 2 & $* * 2$ & 4 & 1 & -7 & 13 & -7 & 1 & & & & \\
\hline 11 & CYC 60 & $* * 2$ & 4 & 1 & -7 & 14 & -8 & 1 & & & & \\
\hline 12 & CYC 11 & $* * 2$ & 5 & 1 & -9 & 28 & -35 & 15 & -1 & & & \\
\hline 13 & CYC 13 & $* * 2$ & 6 & 1 & -11 & 45 & -81 & 70 & -21 & 1 & & \\
\hline 14 & BETA 3 & $* * 2$ & 8 & 1 & -15 & 83 & -220 & 303 & $-2=0$ & 83 & -15 & 1 \\
\hline 15 & CYC 24 & $* * 2$ & 2 & 1 & -4 & 1 & & & & & & \\
\hline 16 & CYC 20 & $* * 2$ & 2 & 1 & -5 & 5 & & & & & & \\
\hline 17 & CYC 36 & $* * 2$ & 3 & 1 & -6 & 9 & -3 & & & & & \\
\hline 18 & CYC 28 & $* * 2$ & 3 & 1 & -7 & 14 & -7 & & & & & \\
\hline 19 & CYC 60 & $*-2$ & 4 & 1 & -8 & 14 & -7 & 1 & & & & \\
\hline 20 & CYC 15 & $* * 2$ & 4 & $i$ & -9 & 26 & -24 & 1 & & & & \\
\hline 21 & SALEMI & +2 & 5 & 1 & -9 & 27 & -31 & 12 & -1 & & & \\
\hline 22 & 5o & $* * 2$ & 5 & 1 & -10 & 33 & -40 & 16 & -1 & & & \\
\hline 23 & $5 c$ & $* * 2$ & 5 & 1 & -11 & 43 & -71 & 42 & -1 & & & \\
\hline 24 & $5 i$ & $* * 2$ & 5 & 1 & -11 & 43 & -72 & 49 & -9 & & & \\
\hline 25 & $6 \mathrm{~m}$ & $* * 2$ & 6 & 1 & -11 & 43 & -72 & 51 & -14 & 1 & & \\
\hline 23 & CYC 84 & $* * 2$ & 6 & 2 & -11 & 44 & -78 & 60 & -16 & 1 & & \\
\hline 27 & $6 f$ & $* * 2$ & 6 & 1 & -13 & 64 & -147 & 153 & -54 & 1 & & \\
\hline 28 & $7 f$ & $* * 2$ & 7 & 1 & -13 & 64 & $-15 ?$ & 182 & -104 & 24 & -1 & \\
\hline 29 & 80 & $* * 2$ & 8 & 1 & -15 & 89 & -269 & 441 & -383.3 & 1.58 & -24 & 1 \\
\hline 30 & CYC 17 & $* * 2$ & 8 & 1 & -15 & 91 & -286 & 495 & -462 & 210 & -36 & 1 \\
\hline 31 & CYC1:0 & +2 & 8 & 1 & -16 & 105 & $-3 i 4$ & 714 & -7234 & 440 & -96 & 1 \\
\hline
\end{tabular}
$\theta+\theta^{-1}+2$, where $\theta$ is the smallest known Salem number (see Boyd [1]) and CYC n $* * 2=(2 \cos 2 \pi / n)^{2}$. 
The 31 polynomials $P_{j}$ finally used in the computation are given in Table 3 . The table of optimal $c_{j}$ 's for each of the 54 runs has not been included in the paper, but is obtainable from the author. For example, in Run $1, p / 2=0.05$ and

$$
\begin{aligned}
g(x, \mathbf{c})= & x^{0.05}-0.047912906 \log x-0.001244003 \log |x-1| \\
& -0.000004535 \log |x-2| \\
& -\cdots-0.000040703 \log \left|x^{4}-8 x^{3}+14 x^{2}-7 x+1\right|
\end{aligned}
$$

is the function whose minimum for $x>0$ is $(1.01822)^{0.1}$, this value being read from Run 1 of Table 1.

For the purpose of proving Theorem 1, how the $c_{j}$ 's were obtained is irrelevant. All that is necessary is to verify that, for the given $c_{j}$ 's for a particular run, the function

$$
g(x, \mathbf{c})=x^{p / 2}-\sum c_{j} \log \left|P_{j}(x)\right|
$$

has its minimum at or above $\left(\mathrm{MBOUND}_{p}\right)^{p}$, where $\mathrm{MBOUND}_{p}$ is given by the corresponding run of Table 1 . This can be done by a straightforward program which uses calculus to find the local minima of $g(x, \mathbf{c})$, for $\mathbf{c}$ fixed.

For Theorem 2, we use the results of Table 1, combined with the following result (following McAuley):

LemMA $1\left(\left[6\right.\right.$, p. 26] or $\left[10\right.$, p. 76]). For fixed $x_{1}, x_{2}, \ldots, x_{d} \geqslant 0,\left(\frac{1}{d} \sum_{i=1}^{d} x_{i}^{p}\right)^{1 / p}$ is an increasing function of $p$, for $0<p<\infty$.

From the lemma it follows that for fixed $\alpha, M_{p}(\alpha)$ is an increasing function of $p$, and also easily that $a_{p}$ and $c_{p}$ defined by $(0.2-0.3)$, are increasing functions of $p$.

The values of $p$ in Table 1 are chosen so that, if for two consecutive runs we have

$\begin{array}{ccccc}\text { Run } \# & p & \text { MBOUND } & \text { NMEAS } & \text { NMUSE } \\ i & p_{i} & b_{i} & m_{i} & n_{i} \\ i+1 & p_{i+1} & b_{i+1} & m_{i+1} & n_{i+1}\end{array}$

then at $p=p_{i+1}$, the smallest $n_{i}$ measures are all $<b_{i}$. Now suppose for $p \in$ $\left[p_{i}, p_{i+1}\right]$, there is an $\alpha$ with $M_{p}(\alpha)<b_{i}$. Then by Lemma $1, M_{p_{i}}(\alpha)<b_{i}$ also, so $\alpha$ must be one of those with $M_{p_{i}}(\alpha)<b_{i}$, i.e. $\alpha$ must be a zero of one of the $m_{i}$ polynomials whose polynomial numbers appear on row $i$. The number $p_{i+1}$ is simply chosen as the largest $p$, with three digits after the decimal point, such that $n_{i}$ of these $m_{i}$ measures $M_{p}(\alpha)$ remain less than $b_{i}$. In principle it is possible to take $n_{i}=m_{i}$ (i.e. NMEAS $=$ NMUSE). However, when this is done $p_{i+1}$ may be only slightly larger than $p_{i}$, so that a very large number of runs would be required to cover $[.1,30]$. In practice, therefore, NMUSE was usually chosen smaller than NMEAS, in order to keep the amount of computation reasonable. It is this that makes the numbers of isolated points obtained in Theorem 2 generally smaller than the numbers obtained in Theorem 1. NMUSE is the smallest number of isolated points in the interval concerned. For $p$ towards the left of the interval, the number of isolated points may be larger. Note also that some values of $p$ are chosen to be smaller than necessitated by the above discussion, so that results for particular round values of $p$, e.g. $p=1$, $p=2$, etc., are shown. 
Let us now look briefly at how, for a fixed $p$, the results obtained could possibly be improved. Look, for instance, at the solution to (1.2) obtained for $p=1$ (Table 4). The optimal $\mu$ is an atomic measure, with weights at the following points:

TABLE 4

The optimal (atomic) measure for $p=1$.

$\begin{array}{ll}\text { X-VALUE } & \text { WEIGHT } \\ 0.13121 & 0.06534 \\ 0.13313 & 0.09436 \\ 0.30190 & 0.10743 \\ 0.46361 & 0.03007 \\ 0.59601 & 0.13408 \\ 1.40390 & 0.07690 \\ 1.70937 & 0.11160 \\ 2.13317 & 0.10593 \\ 3.00562 & 0.04129 \\ 3.65058 & 0.12435 \\ 4.84044 & 0.09375 \\ 4.88644 & 0.01490\end{array}$

From this measure it may be possible to guess a polynomial (which of course corresponds to an atomic measure with all nonzero weights equal) for which $M_{p}(\alpha)$ is the smallest measure of an $\alpha$ with $\alpha^{2}$ 'not on the list'. However, there seems to be a limit to how effective this method will be. One reason for this may be that there seems to be no way of making use of the fact that the nonzero weights of measures corresponding to polynomials are all equal. It may be necessary to use constraints other than the resultant constraints (1.1) we have used. For example, the discriminant constraints

$$
\prod_{i \neq j}\left|\alpha_{i}-\alpha_{j}\right| \geqslant 1
$$

were used by Siegel [13] and later authors. Were we able to somehow use the constraints

$$
\left\{\begin{array}{l}
\prod_{i, j}\left|\alpha_{i}^{2}-\alpha_{j}-2\right| \geqslant 1, \\
\prod_{i, j}\left|\alpha_{i}^{2}+\alpha_{j}-2\right| \geqslant 1, \\
\prod_{i, j}\left|\alpha_{i}+\alpha_{j}\right| \geqslant 1,
\end{array}\right.
$$

we could exclude all $\alpha$ of the form $2 \cos 2 \pi / n$. Also, we could exclude all the $\beta_{j}$ by the constraint

$$
\prod_{i, j}\left|\alpha_{i} \alpha_{j}+1\right| \geqslant 1
$$

and perhaps all fixed points of iterates of $H$ by using, for $\varepsilon= \pm 1$,

$$
\prod_{i, j}\left|\alpha_{i}^{2}+\varepsilon \alpha_{i} \alpha_{j}-1\right| \geqslant 1 .
$$

It is then conceivable that (somehow?) one could show that, apart from the $M_{p}(\alpha)$ of the $\alpha$ 's mentioned just above, $M_{p}(\alpha)$ would be $\geqslant \min \left(a_{p}, c_{p}\right)$. The basic problem with such an approach is that these constraints translate into constraints quadratic 
in $\mu$-for instance (1.10) becomes

$$
\int_{0}^{\infty} d \mu_{\alpha}(x) \int_{0}^{\infty} d \mu_{\alpha}(y) \log \left|x^{2}+\varepsilon x y-1\right| \geqslant 0 .
$$

It is not clear to me how a minimization problem with such constraints could be successfully tackled numerically.

2. Everywhere Denseness in $\left(a_{p}, \infty\right)$. Let $F_{n}(x)$ be the distribution function of the absolute values of the conjugates of $\beta_{n}$ :

$$
F_{n}(x)=2^{-n} \times \# \text { of conjugates of } \beta_{n} \text { in }[-x, x]
$$

(see [14, Section 3]). When both the integral and the limit exist, put

$$
a(g)=\lim _{n \rightarrow \infty} \int_{0}^{\infty} g(x) d F_{n}(x)
$$

for a given function $g:[0, \infty) \rightarrow \mathbf{R}$. Then $a_{p}=\left(a\left(x^{p}\right)\right)^{1 / p}$. Let $\mathscr{T}(g)$ be the set of all means

$$
M_{g}(\alpha)=d(\alpha)^{-1} \sum_{i=1}^{d(\alpha)} g\left(\left|\alpha_{i}\right|\right)
$$

for $\alpha$ a totally real algebraic integer, with conjugates $\alpha=\alpha_{1}, \ldots, \alpha_{d(\alpha)}$. Then $\mathfrak{N}_{p}=\left\{x^{1 / p}: x \in \mathfrak{M}\left(x^{p}\right)\right\}$. Note also that

$$
M_{g}\left(\beta_{n}\right)=\int_{0}^{\infty} g(x) d F_{n}(x) .
$$

In this section we prove

TheOREM 3. Let $g: \mathbf{R}_{+} \rightarrow \mathbf{R}_{+}$be a monotonic increasing function, zero on $[0,1]$, such that

$$
\lim _{x \rightarrow \infty} g(x+1) / g(x)=1
$$

and

the values of $\log _{2}^{\prime} g(2 k+1) \bmod 1(k=0,1,2, \ldots)$ are everywhere dense in $(0,1)$.

Then the limit $a(g)$ exists, and $\mathscr{N}(g)$ is dense in $(a(g), \infty)$.

COROllary 3. The set $\mathfrak{T}(g)$ is dense in $(a(g), \infty)$ for $p>0$ fixed and

(1) $g(x)=x^{p}$

(2) $g(x)=\left(\log _{+} x\right)^{p}$.

(3) $g(x)=|\log x|^{p}$.

As in [14], we need to define $\beta_{0}^{(b)}=b$, where $b$ is an odd positive integer, and $\beta_{n}^{(b)}$ $(n \geqslant 1)$ by $\beta_{n}^{(b)}>1$ and

$$
\beta_{n}^{(b)}-\left(\beta_{n}^{(b)}\right)^{-1}=\beta_{n-1}^{(b)} .
$$

Let $\beta_{n}^{(b)}$ have conjugates of absolute values $\beta_{n, i}^{(b)}\left(i=1,2, \ldots, 2^{n}\right)$, with $\beta_{n, 1}^{(b)}=\beta_{n}^{(b)}$. Put $\beta_{n, i}=\beta_{n, i}^{(1)}$ and note that $\beta_{n}=\beta_{n}^{(1)}$. Let

$$
\mathscr{B}_{n}^{(b)}=\left\{\beta_{n, i}^{(b)} \mid i=1,2, \ldots, 2^{n}\right\}
$$


and $\mathscr{G}_{n}=\mathscr{G}_{n}^{(1)}$. First we need

LEMMA 2. The elements of $\mathscr{B}_{n}^{(b)}$ alternate with those of $\{0, \infty\} \cup \mathscr{R}_{0} \cup \mathscr{B}_{1} \cup \cdots \cup$ $\Re_{n-1}$ on the nonnegative half-line.

Proof. We shall actually prove more: that

(1) The elements of $\mathscr{B}_{n}$ alternate with $\{0, \infty\} \cup \mathscr{B}_{0} \cup \mathscr{G}_{1} \cup \cdots \cup \mathscr{B}_{n-1}\left(=\mathscr{U}_{n}\right.$ say), and

(2) Suppose we are given three consecutive elements of $\mathscr{q}_{n} \cup \mathscr{B}_{n}, u_{1}<\beta_{n, i}<u_{2}$ with $\beta_{n, i} \in \mathscr{H}_{n}$, one of $u_{1}$ and $u_{2}$ in $\mathscr{U}_{n-1}$, the other in $\mathscr{H}_{n-1}$. Then for all $b>1$, and the same $n, i$, the elements $\beta_{n, i}^{(b)}$ all lie in

$$
\begin{cases}\left(u_{1}, \beta_{n, i}\right) & \text { if } u_{1} \in \mathcal{Q}_{n-1}, \\ \left(\beta_{n, i}, u_{2}\right) & \text { if } u_{2} \in \mathcal{Q}_{n-1} .\end{cases}
$$

The truth of these statements follows by induction on $n$. They are true for $n=1$ since $U_{0}=\{0, \infty\}$ and

$$
0<\left(\beta_{1}^{(b)}\right)^{-1}<\beta_{1}^{-1}<\beta_{0}=1<\beta_{1}<\beta_{1}^{(b)}<\infty .
$$

Now define $H$ and $H^{-1}$ by

$$
H x=x-x^{-1}
$$

and

$$
H^{-1} x=\frac{1}{2}\left(x+\left(x^{2}+4\right)^{1 / 2}\right)
$$

as in [14]. Then, assuming the truth of (1) and (2) for $n$, we can prove them for $n+1$, using the result that, from (2.7),

$$
\mathscr{B}_{n+1}^{(b)}=H^{-1} \mathscr{B}_{n}^{(b)} \cup\left(H^{-1} \mathscr{B}_{n}^{(b)}\right)^{-1}
$$

and the fact that $H^{-1}$ preserves order on $(0, \infty)$.

Let $F(x)$ be the continuous function defined in [14], and satisfying

$$
F(x)=\lim _{n \rightarrow \infty} F_{n}(x) \text {. }
$$

LemMA 3. Let $g:[1, \infty) \rightarrow \mathbf{R}_{+}$be monotonic increasing, such that a $(g)$ exists, and such that

$$
g(x)=O\left(A^{x^{2}}\right) \text { for some } A: 1<A<\sqrt{2} .
$$

Then $a(g)$ is finite, and

$$
a(g)=\lim _{n \rightarrow \infty} \int_{1}^{\infty} g(x) d F_{n}(x)
$$

Proof. We have, for some constant $A_{2}$,

$$
\left|\int_{\beta_{n}}^{\beta_{n+1}} g d F\right| \leqslant \sup _{x \in\left[\beta_{n}, \beta_{n+1}\right]}|g| \int_{\beta_{n}}^{\beta_{n+1}} d F<A_{2} A^{\beta_{n+1}^{2} 2^{-n}}
$$

using the fact [14, Lemma 7] that $\int_{\beta_{n}}^{\beta_{n+1}} d F=2^{-n-2}$. Since, again from [14, Eq. (5.1)]

$$
b \leqslant \beta_{n}^{(b)} \leqslant \sqrt{2 n+b^{2}}<b+n / b,
$$


$\beta_{n+1}^{2} \leqslant 2 n+3$, and hence from (2.15),

$$
\left|\int_{\beta_{n}}^{\beta_{n+1}} g d F\right| \leqslant A_{2} A^{3}\left(A^{2} / 2\right)^{n} .
$$

Since $A^{2}<2$, the whole integral $\int_{1}^{\infty}=\sum_{n=0}^{\infty} \int_{\beta_{n}}^{\beta_{n+1}}$ is finite.

To prove (2.14), note that, from [14, Lemma 7],

$$
\int_{\beta_{n, l+1}}^{\beta_{n, t}} d F=2^{-n}
$$

and so, since $g$ is assumed to be monotonic, $\int_{\beta_{n, i+1}}^{\beta_{n, 1}} g d F$ lies between $2^{-n} g\left(\beta_{n, i+1}\right)$ and $2^{-n} g\left(\beta_{n, i}\right)$. Since $\int_{\beta_{n, l+1}+}^{\beta_{n, l}+} g d F_{n}=2^{-n} g\left(\beta_{n, i}\right)$, we have

$$
0<\int_{\beta_{n, i+1}+}^{\beta_{n, i}+} g d\left(F_{n}-F\right)<2^{-n}\left(g\left(\beta_{n, i}\right)-g\left(\beta_{n, i+1}\right)\right) .
$$

Now let $e=2^{n-1}+1$, so that $\beta_{n, e}<1<\beta_{n, e-1}$. Then, from (2.19), (2.16) and (2.13),

$$
\int_{\beta_{n, e^{+}}}^{\beta_{n}+} g d\left(F_{n}-F\right)<2^{-n} g\left(\beta_{n}\right)<A_{3}\left(A^{2} / 2\right)^{n} .
$$

Since $g=0$ for $x<1, \int_{\beta_{n, 0}}^{1} g d\left(F_{n}-F\right)=0$, and trivially $\int_{\beta_{n}+}^{\infty} g d F_{n}=0$, and from (2.17), $\int_{\beta_{n}+}^{\infty} g d F<A_{4}\left(A^{2} / 2\right)^{n}$. Thus $\left|\int_{1}^{\infty} g d\left(F_{n}-F\right)\right|<A_{5}\left(A^{2} / 2\right)^{n} \rightarrow 0$ as $n \rightarrow \infty$.

We can now prove Theorem 3 . The method is essentially that of [14, Theorem 2], except that we need the more detailed information on the position of the $\beta_{n, i}$ provided by Lemma 2.

Let $g$ be as in the statement of the theorem, and $r>a(g)$ and $\varepsilon>0$ be given. We shall exhibit an odd integer $b$, and an $N$ such that

$$
\left|M_{g}\left(\beta_{n}^{(b)}\right)-r\right|<\varepsilon
$$

for infinitely many $n \geqslant N$. The idea of the proof is that the conjugates of $\beta_{n}^{(b)}$ are distributed on the real line almost exactly as the conjugates of $\beta_{n}$ are, apart from the largest conjugate of $\beta_{n}^{(b)}, \beta_{n}^{(b)}$ itself. The values of $n$ and $b$ are chosen so that the $2^{-n} \beta_{n}^{(b)}$ term in $M_{g}\left(\beta_{n}^{(b)}\right)$ makes $M_{g}\left(\beta_{n}^{(b)}\right)$ approximate $r$.

From Lemma 3, we have

$$
M_{g}\left(\beta_{j}\right)=\left(1-\varepsilon_{j}\right) a(g),
$$

where $\varepsilon_{j} \rightarrow 0$ as $j \rightarrow \infty$. Then using Lemma 2,

$$
\begin{aligned}
M_{g}\left(\beta_{n}^{(b)}\right) & =2^{-n} \sum_{i=1}^{2^{n}} g\left(\beta_{n, i}^{(b)}\right) \geqslant 2^{-n}\left(g\left(\beta_{n}^{(b)}\right)+\sum_{x \in \mathcal{Q}_{n} \backslash\left\langle\infty, \beta_{n-1}\right\}} g(x)\right) \\
& =2^{-n}\left(g(0)+\sum_{j=0}^{n-1} \sum_{i=1}^{2^{j}} g\left(\beta_{j, i}\right)-g\left(\beta_{n-1}\right)+g\left(\beta_{n}^{(b)}\right)\right) \\
& =\sum_{j=0}^{n-1} 2^{-(n-j)}\left(1-\varepsilon_{j}\right) a(g)+2^{-n}\left(g\left(\beta_{n}^{(b)}\right)-g\left(\beta_{n-1}\right)\right) \\
& \geqslant a(g)+2^{-n}\left(g\left(\beta_{n}^{(b)}\right)-g\left(\beta_{n-1}\right)-a(g)\right)-T_{n},
\end{aligned}
$$

where $T_{n}=a(g) \sum_{j=0}^{n-1} 2^{-(n-j)}\left|\varepsilon_{j}\right| \rightarrow 0$ as $n \rightarrow \infty$. 
Similarly, in the other direction,

$$
\begin{aligned}
M_{g}\left(\beta_{n}^{(b)}\right) & \leqslant \sum_{j=1}^{n-1} 2^{-(n-j)} M_{g}\left(\beta_{j}^{(b)}\right)+2^{-n} g\left(\beta_{n}^{(b)}\right) \\
& =\left(1-2^{-n}\right) a(g)+T_{n}+2^{-n}\left(\beta_{n}^{(b)}\right) .
\end{aligned}
$$

Combining (2.23) and (2.24), and using (2.16)

$$
\left|M_{g}\left(\beta_{n}^{(b)}\right)-\left(a(g)+2^{-n} g\left(\beta_{n}^{(b)}\right)\right)\right| \leqslant T_{n}+2^{-n}(a(g)+g(\sqrt{2 n-1})) .
$$

Since the right-hand side tends to 0 as $n \rightarrow \infty$, we have for $n>N$, say,

$$
\left|M_{g}\left(\beta_{n}^{(b)}\right)-\left(a(g)+2^{-n} g\left(\beta_{n}^{(b)}\right)\right)\right|<\varepsilon / 3 .
$$

The next task is to arrange for $a(g)+2^{-n} g\left(\beta_{n}^{(b)}\right)$ to be close to $r$. From (2.6) we can choose increasing sequences $\left\{n_{i}\right\},\left\{b_{i}\right\}$ of integers, with the $b_{i}$ odd, such that

$$
\left|\log _{2}\left(g\left(b_{i}\right)\right)-\log _{2}(r-a(g))-n_{i}\right|<\log _{2}\left(1+\frac{\varepsilon}{3(r-a(g))}\right),
$$

from which we readily get

$$
\left|2^{-n_{i}} g\left(b_{i}\right)-(r-a(g))\right|<\varepsilon / 3 \text {. }
$$

Finally, it remains only to estimate $2^{-n}\left(g\left(\beta_{n}^{(b)}\right)-g(b)\right)$ for $n=n_{i}, b=b_{i}$. To do this, we note that (2.5) implies easily that $\log (g(b))=o(b)$, so that $n_{i}=o\left(b_{i}\right)$ from (2.27), i.e. $n_{i} / b_{i} \rightarrow 0$ as $i \rightarrow \infty$. Now from (2.16), $g\left(\beta_{n}^{(b)}\right)<g(b+1)$ for $b=b_{i}$, and $i$ sufficiently large, and so

$$
2^{-n}\left(g\left(\beta_{n}^{(b)}\right)-g(b)\right) \leqslant 2^{-n} g(b)(g(b+1) / g(b)-1) \rightarrow 0
$$

for $n=n_{i}, b=b_{i}, i \rightarrow \infty$, using (2.28) and (2.5). Hence we can choose an $I_{1}$ such that

$$
2^{-n}\left(g\left(\beta_{n}^{(b)}\right)-g(b)\right)<\varepsilon / 3 \text { for } n=n_{i}, b=b_{i}, i>I_{1} .
$$

Now, combining (2.26), (2.28) and (2.29),

$$
\left|M_{g}\left(\beta_{n}^{(b)}\right)-r\right|<\varepsilon \text { for } n=n_{i}, b=b_{i}, i>I_{1},
$$

provided also that $n>N_{1}$. This proves the theorem.

Proof of Corollary 3. We will need the fact [14, Eq. (3.4)] that $F(x)+F\left(x^{-1}\right)=1$, so that

$$
d F\left(x^{-1}\right)=-d F(x) .
$$

For later use also note that [14, Eqs. (3.5), (3.6)]

$$
F(x)= \begin{cases}\frac{1}{2}\left(1+F\left(x-x^{-1}\right)\right), & x \geqslant 1, \\ \frac{1}{2}\left(1-F\left(x^{-1}-x\right)\right), & 0 \leqslant x \leqslant 1 .\end{cases}
$$

Let $p>0$ be fixed. The result for $\left(\log _{+} x\right)^{p}$ is immediate from the theorem, since this function is monotonic, and zero on $[0,1]$. Now let $g(x)=x^{p}$. Then, since 
$\left( \pm \beta_{n, i}^{(b)}\right)^{-1}$ is a conjugate of $\beta_{n, i}^{(b)}( \pm$ denoting ' + or - ', not ' + and - '),

$$
\begin{aligned}
g\left(\beta_{n}^{(b)}\right) & =2^{-n} \sum_{i=1}^{2^{n}}\left(\beta_{n, i}^{(b)}\right)^{p} \\
& =2^{-n} \sum_{i=1}^{2^{n-1}}\left(\left(\beta_{n, i}^{(b)}\right)^{p}+\left(\beta_{n, i}^{(b)}\right)^{-p}\right)=M_{g^{*}}\left(\beta_{n}^{(b)}\right),
\end{aligned}
$$

where

$$
g^{*}(x)= \begin{cases}x^{p}+x^{-p}, & x>1 \\ 0, & 0 \leqslant x \leqslant 1\end{cases}
$$

Since $g^{*}$ is increasing, and 0 on $[0,1]$, we can apply Theorem 3 to it. The values of $M_{g}\left(\beta_{n}^{(b)}\right)$ are therefore dense on $\left(a\left(g^{*}\right), \infty\right)$. Further,

$$
a\left(g^{*}\right)=\int_{1}^{\infty}\left(x^{p}+x^{-p}\right) d F(x)=\int_{0}^{\infty} x^{p} d F(x)=a(g)
$$

using (2.31), which proves the corollary for $g(x)=x^{p}$.

Now put $g(x)=|\log x|^{p}$. Then it is easily shown that $M_{g}\left(\beta_{n}^{(b)}\right)=2 M_{g}\left(\beta_{n}^{(b)}\right)$, where $g^{+}(x)=\left(\log _{+} x\right)^{p}$, and that $a(g)=2 a\left(g^{+}\right)$. Hence, applying Theorem 3 to $g^{+}$, we have the values of $M_{g}\left(\beta_{n}^{(b)}\right)$ dense on $(a(g), \infty)$.

3. Everywhere Denseness in $\left(c_{p}, \infty\right)$. We prove

THEOREM 4. Let $g: \mathbf{R}_{+} \rightarrow \mathbf{R}_{+}$be a function such that

$$
\lim _{x \rightarrow \infty} g(x)=\infty
$$

and which satisfies a Lipschitz condition

$$
|g(x)-g(y)|<B(\lambda)|x-y|
$$

for $x, y \in[0, \lambda]$, for each $\lambda>0$. Then $\mathfrak{K}_{g}$ is dense on $(c(g), \infty)$, where

$$
c(g)=\frac{2}{\pi} \int_{0}^{\pi / 2} g(2 \cos \theta) d \theta
$$

Corollary 4. $\Re_{p}$ is dense on $\left(c_{p}, \infty\right)$, where $c_{p}=\left(c\left(x^{p}\right)\right)^{1 / p}$.

The proof is basically an extension of the proof of Robinson [11, p. 309] showing that for each $\varepsilon>0$ the interval $[-2-\varepsilon, 2+\varepsilon]$ contains infinitely many conjugate sets of algebraic integers; see also Ennola [2]. Robinson's result is essentially Corollary 4 above for $p=\infty$. His basic lemma can be stated as

LEMMA 4. Given a rational number $\lambda>1$, there is an infinite sequence $\left\{n_{i}\right\}$ of increasing even integers and corresponding totally real algebraic integers $\alpha^{(i)}(i=$ $1,2, \ldots)$ with $\operatorname{deg} \alpha^{(i)}=n_{i}$ whose conjugates $\alpha_{j}^{(i)}\left(j=1, \ldots, n_{i}\right)$ satisfy

$$
2 \lambda \cos (j \pi / n)<\alpha_{j}^{(i)}<2 \lambda \cos ((j-1) \pi / n) \quad(j=1,2, \ldots, n) .
$$

Here $n=n_{i}$.

For convenience define

$$
g_{n, \lambda}=\frac{2}{n} \sum_{j=1}^{n / 2} g(2 \lambda \cos (j \pi / n)),
$$




$$
g_{\lambda}=\frac{2}{\pi} \int_{0}^{\pi / 2} g(2 \lambda \cos \theta) d \theta
$$

Then one has easily

LEMMA 5. For $g$ as in Theorem 4,

$$
g_{\lambda}=\lim _{n \rightarrow \infty} g_{n, \lambda} .
$$

Further both $g_{n, \lambda}$ and $g_{\lambda}$ are continuous functions of $\lambda$, and $g_{\lambda}$ tends to $\infty$ as $\lambda \rightarrow \infty$.

Proof of Theorem 4. Let $r>c(g)$ and $\varepsilon>0$ be given. By Lemma 5 there is a real $\lambda_{1}>1$ such that

$$
g_{\lambda_{1}}=r,
$$

and by the same lemma we can choose $N$ such that

$$
\left|g_{n, \lambda_{1}}-g_{\lambda_{1}}\right|<\varepsilon / 3
$$

for $n>N$. By the continuity of $g_{n, \lambda}$ as a function of $\lambda$, we can choose $\lambda>1$ rational and such that

$$
\left|g_{n, \lambda}-g_{n, \lambda_{1}}\right|<\varepsilon / 3 .
$$

Let $B=B(2 \lambda+2)$, as in (3.2), and choose $\alpha^{(i)}$ as in Lemma 4. Then from (3.4), for $n=n_{i}$,

$$
\begin{array}{r}
0<\alpha_{j}^{(i)}-2 \lambda \cos (j \pi / n)<2 \lambda(\cos ((j-1) \pi / n)-\cos (j \pi / n)) \\
\quad(j=1, \ldots, n) .
\end{array}
$$

Hence, by (3.2),

$$
\begin{aligned}
\left|\frac{1}{n} \sum_{j=1}^{n} g\left(\left|\alpha_{j}^{(i)}\right|\right)-g_{n, \lambda}\right| & \leqslant \frac{1}{n} \sum_{j=1}^{n}\left|g\left(\left|\alpha_{j}^{(i)}\right|\right)-g(|2 \lambda \cos (j \pi / n)|)\right| \\
& \leqslant \frac{2 \lambda B}{n} \sum_{j=1}^{n}(\cos ((j-1) \pi / n)-\cos (j \pi / n)) \\
& =\frac{4 \lambda B}{n}<\varepsilon / 3
\end{aligned}
$$

for $n>\lceil 12 \lambda B / \varepsilon\rceil=N_{1}$ say. That is, for $n=n_{i}>N$, we have

$$
\left|M_{g}\left(\alpha^{(i)}\right)-g_{n, \lambda}\right|<\varepsilon / 3 .
$$

Hence (3.8), (3.9), (3.10) and (3.11) together give, for $n_{i}>\max \left(N, N_{1}\right)$,

$$
\left|M_{g}\left(\alpha^{(i)}\right)-r\right|<\varepsilon
$$

4. The Case of Small $p$ : $0<p \leqslant 0.1$. The computational methods described in Section 1 did not cover $p$ in the interval $0<p<0.1$, so we now consider this case.

THEOREM 5. For $0<p \leqslant 0.1$, the smallest element of $\mathfrak{M}_{p}$ is $M_{p}\left(\frac{1}{2}(1+\sqrt{5})\right) \simeq 1+$ $0.1158 p$, and all other elements of $\Re_{p}$ lie in $(1+0.1459 p, \infty)$. 
For a given $\alpha$ (with conjugates $\alpha_{i}$ ) and $p$ small

$$
\begin{aligned}
\left(M_{p}(\alpha)\right)^{p} & =\frac{1}{d} \sum_{i=1}^{d} \exp \left(p \log \left|\alpha_{i}\right|\right) \\
& =1+\frac{p}{d} \sum_{i=1}^{d} \log \left|\alpha_{i}\right|+\frac{p^{2}}{2 d} \sum_{i=1}^{d} \log ^{2}\left|\alpha_{i}\right|+\cdots .
\end{aligned}
$$

Now

$$
\frac{1}{d} \sum \log \left|\alpha_{i}\right|=\frac{1}{d} \log \mid \text { Norm } \alpha \mid,
$$

which can be 0 . However, one can show by the methods of Section 1 that $(1 / d) \sum \log ^{2}\left|\alpha_{i}\right|$ is bounded away from 0 (and indeed find its first 3 or 4 isolated values). But this information is not sufficient to bound $M_{p}(\alpha)$ away from 1, as the following example shows (the $x_{i}$ corresponding to $\left.\log \left|\alpha_{i}\right|\right)$ :

Take $x_{1}=x_{2}=\cdots=x_{d-1}=s(d-1)^{-1 / 2}, x_{d}=-s(d-1)^{1 / 2}$. Then $\sum x_{i}=0$, $(1 / d) \sum x_{i}^{2}=s^{2}$, but on taking $d=\lambda^{2} /\left(p^{2} s^{2}\right)$,

$$
\frac{1}{d} \sum_{i} \exp \left(x_{i} p\right)=1+s^{2} p^{2}\left(\frac{1}{\lambda}-\frac{1}{\lambda^{2}}+\frac{e^{-\lambda}}{\lambda^{2}}\right)+o\left(p^{2}\right) .
$$

Thus, by taking $\lambda$ as large as we please, we have shown that $(1 / d) \sum_{i} \exp \left(x_{i} p\right)$ cannot be bounded away from 1 , given $\sum x_{i} \geqslant 0$ and $(1 / d) \sum x_{i}^{2}=s^{2}>0$.

We can however use the fact that $(1 / d) \sum_{i} \log _{+}\left|\alpha_{i}\right|$ is bounded away from 0 to prove Theorem 5. To do this we need the standard

LEMMA $6([6, \mathrm{p} .72])$. If the function $\phi$ is convex upwards, then

$$
\frac{1}{d} \sum_{i=1}^{d} \phi\left(x_{i}\right) \geqslant \phi\left(\frac{1}{d} \sum_{i=1}^{d} x_{i}\right) .
$$

Next, the simple

LEMMA 7. If $\sum_{i=1}^{d} x_{i} \geqslant 0$ and $(1 / d) \sum_{x_{1} \geqslant 0} x_{i}=c$, then

$$
\begin{gathered}
\frac{1}{d} \sum_{x_{i}<0} x_{i} \geqslant-c, \\
\frac{1}{d} \sum_{x_{i} \geqslant 0} x_{i}^{2} \geqslant c^{2}, \\
\frac{1}{d} \sum_{i=1}^{d} \exp \left(x_{i} p\right) \geqslant 1+\frac{1}{2} c^{2} p^{2} \text { for } p>0 .
\end{gathered}
$$

Proof. The first result is immediate. Further, on applying Lemma 6 with $\phi(x)=x^{2}$ to the nonnegative $x_{i}$ 's, padded with an appropriate number of zeros,

$$
\frac{1}{d} \sum_{x_{i} \geqslant 0} x_{i}^{2} \geqslant\left(\frac{1}{d} \sum_{x_{i} \geqslant 0} x_{i}\right)^{2} \geqslant c^{2} .
$$


For (4.5), use the fact that

$$
\exp (x) \geqslant \begin{cases}1+x+\frac{1}{2} x^{2}, & x \geqslant 0 \\ 1+x, & x<0\end{cases}
$$

and (4.4).

We now prove a stronger version of (4.5), with the asymptotically (as $p \searrow 0$ ) best possible coefficient of $p^{2}$. (To see this, take half of the $x_{i}$ equal to $2 c$ and the rest equal to $-2 c$.)

LEMMA 8. If $\sum_{i=1}^{d} x_{i} \geqslant 0,(1 / d) \sum_{x_{1} \geqslant 0} x_{i}=c$ and $0<2 c p<1$, then

$$
\frac{1}{d} \sum_{i=1}^{d} \exp \left(x_{i} p\right) \geqslant 1+2 c^{2} p^{2}-\frac{(2 c p)^{4}}{3\left(1-(2 c p)^{2}\right)^{2}} \text {. }
$$

Proof. Suppose that of the $d x_{i}$ 's, $y d$ are $<0$ and $(1-y) d$ are $\geqslant 0$. Let $k=2 c p<$ 1. Then, applying Lemma 6 to $e^{p x}$ and using (4.3), we get (ignoring the trivial case $y=0$ )

$$
\begin{gathered}
\frac{1}{(1-y) d} \sum_{x_{i} \geqslant 0} \exp \left(x_{i} p\right) \geqslant \exp \left(\frac{1}{2} k /(1-y)\right), \\
\frac{1}{y d} \sum_{x_{l}<0} \exp \left(x_{i} p\right) \geqslant \exp \left(-\frac{1}{2} k / y\right) .
\end{gathered}
$$

Hence

(4.8) $\frac{1}{d} \sum_{i=1}^{d} \exp \left(x_{i} p\right) \geqslant(1-y) \exp \left(\frac{1}{2} k /(1-y)\right)+y \exp \left(-\frac{1}{2} k / y\right)=f(y)$

say. We now estimate the minimum of $f(y)$ in $(0,1)$. We have

$$
\begin{aligned}
f^{\prime}(y)= & \left(1+\frac{1}{2} k / y\right) \exp \left(-\frac{1}{2} k / y\right)+\left(-1+\frac{1}{2} k /(1-y)\right) \\
& \times \exp \left(\frac{1}{2} k /(1-y)\right)
\end{aligned}
$$

and it is easily checked that $f^{\prime \prime}(y)>0$ in $(0,1)$. So $f$ has at most one minimum in $(0,1)$, with $f(0+)=\exp \left(\frac{1}{2} k\right)$, and $f(1-)=\infty$. We shall show that $f$ does in fact have a minimum, and it occurs for $y$ between $\frac{1}{2}(1-k)$ and $\frac{1}{2}$.

Firstly $f^{\prime}\left(\frac{1}{2}\right)=(1+k) \exp (-k)-(1-k) \exp (k)>0$, as $(1+k) /(1-k)>$ $\exp (2 k)$ for $0<k<1$. Next, put $y=\frac{1}{2}(1-k)$. Then

$$
\begin{aligned}
\frac{1+\frac{1}{2} k / y}{1-\frac{1}{2} k /(1-y)} & =\frac{1+k}{1-k}=\exp \left(2\left(k+k^{3} / 3+k^{5} / 5+\cdots\right)\right) \\
& \leqslant \exp \left(2\left(k+k^{3}+\cdots\right)\right)=\exp \left(2 k /\left(1-k^{2}\right)\right),
\end{aligned}
$$

which shows that $f^{\prime}\left(\frac{1}{2}(1-k)\right)<0$.

Suppose the minimum occurs at $y=\frac{1}{2}(1-\delta)$, where $0<\delta<k$. Then, since for all $x$

$$
\exp (x) \geqslant 1+x+x^{2} / 2+x^{3} / 6
$$


we have

$$
\begin{aligned}
f\left(\frac{1}{2}(1-\delta)\right) & =\frac{1}{2}((1-\delta) \exp (-k /(1-\delta))+(1+\delta) \exp (k /(1+\delta))) \\
& \geqslant 1+\frac{1}{2} k^{2} /\left(1-\delta^{2}\right)-k^{3} \delta /\left(3\left(1-\delta^{2}\right)^{2}\right) \\
& \geqslant 1+\frac{1}{2} k^{2}-k^{4} /\left(3\left(1-k^{2}\right)^{2}\right)
\end{aligned}
$$

We can now prove the theorem. From [15, Eq. (9)] we know that, with four exceptions,

$$
\frac{1}{d(\alpha)} \sum_{i} \log _{+}\left|\alpha_{i}\right| \geqslant \log (1.31040)=0.2703 \text {. }
$$

We can thus apply Lemma 8 with $c \geqslant c_{0}=0.2703$. Since the function $1+x / 2-$ $x^{2} / 3(1-x)^{2}$ is increasing for $0<x<\frac{1}{4}$, it follows from (4.7) that, for $(2 c p)<\frac{1}{2}$, with at most four exceptions

$$
M_{p}(\alpha)^{p}=\frac{1}{d} \sum_{i}\left|\alpha_{i}\right|^{p} \geqslant 1+2\left(c_{0} p\right)^{2}-\frac{\left(2 c_{0} p\right)^{4}}{3\left(1-\left(2 c_{0} p\right)^{2}\right)^{2}} .
$$

However if $2 c p \geqslant \frac{1}{2}$, then from (4.5)

$$
M_{p}(\alpha)^{p} \geqslant \frac{33}{32}=1.03125 \geqslant 1+0.3125 p \text { for } 0<p \leqslant 0.1 .
$$

Hence we can assume $2 c p<\frac{1}{2}$, and then from (4.10)

$$
\begin{aligned}
M_{p}(\alpha)^{p} & \geqslant 1+p^{2}\left(0.1461-\frac{0.08541 p^{2}}{3\left(1-0.2922 p^{2}\right)^{2}}\right) \\
& \geqslant 1+0.1458 p^{2} \quad \text { for } 0<p \leqslant 0.1 .
\end{aligned}
$$

Then, as $(1+x)^{r}>1+r x$ for $r>1$,

$$
M_{p}(\alpha) \geqslant\left(1+0.1458 p^{2}\right)^{1 / p}>1+0.1458 p \text {. }
$$

Of the four exceptions to which (4.11) and (4.12) do not apply, calculation reveals that it is only for $\alpha= \pm \frac{1}{2}(1 \pm \sqrt{5})$ that (4.12) is actually violated.

5. The Limit Points $a_{p}$ and $c_{p}$. The limit point $c_{p}$ is easy to evaluate from its definition (0.3), giving

$$
c_{p}=2\left(\frac{2}{\pi} \int_{0}^{\pi / 2}(\cos \theta)^{p} d \theta\right)^{1 / p}=2\left[\Gamma\left(\frac{1}{2}(p+1)\right) \pi^{-1 / 2} / \Gamma\left(1+\frac{1}{2} p\right)\right]^{1 / p}
$$

by $[4$, Section 3.261$]$.

Further, as $p \rightarrow 0$

$$
c_{p}=\left(1+\frac{p^{2}}{2} \cdot \frac{2}{\pi} \int_{0}^{\pi / 2} \log ^{2}(2 \cos \theta) d \theta+\cdots\right)^{1 / p}=1+\left(\frac{\pi^{2}}{24}\right) p+\cdots
$$

(see Lewin [8, p. 298, Eq. (40)]). However, note that the same formula is misprinted on p. 170, although it is correct in the first edition).

For small $p$,

$$
a_{p}=\left(1+\frac{p^{2}}{2} \int_{0}^{\infty}(\log x)^{2} d F(x)+\cdots\right)^{1 / p}=1+0.19233 p+\cdots
$$


on computation, so that $a_{p}<c_{p}$ for $p$ small enough. We show below that $a_{2}=c_{2}$ $=\sqrt{2}$, and $a_{p} \rightarrow \infty$ as $p \rightarrow \infty$, while $c_{p} \rightarrow 2$ as $p \rightarrow \infty$. We have found no formula for $a_{p}$ corresponding to (5.2) for $c_{p}$. However, we have obtained a recurrence relation which enables us to recursively evaluate $a_{p}$ for $p$ an even integer. In fact

THEOREM 6. Defining $a_{0}^{0}=1$ we have

$$
a_{2 k}^{2 k}=2 \sum_{j=0}^{k-1} \frac{k}{k+j}\left(\begin{array}{l}
k+j \\
k-j
\end{array}\right) a_{2 j}^{2 j} \quad(k=1,2, \ldots)
$$

so that $a_{2}^{2}=2, a_{4}^{4}=10, a_{6}^{6}=80, a_{8}^{8}=874$, and so on. Further

$$
a_{2 k}>\sqrt{(2 k / e)} \quad(k=1,2, \ldots) .
$$

Proof. Putting $y=x-x^{-1}$ into (2.32), we get

$$
d F(y)=2 d F\left(\frac{1}{2}\left(y+\sqrt{\left(y^{2}+4\right)}\right)\right), \quad y \geqslant 0,
$$

and putting $y=x^{-1}-x$ into (2.33),

$$
d F(y)=-2 d F\left(\frac{1}{2}\left(\sqrt{\left(y^{2}+4\right)}-y\right)\right), \quad y \geqslant 0 .
$$

Hence

$$
\begin{aligned}
a_{p}^{p}= & \left(\int_{0}^{1}+\int_{1}^{\infty}\right) x^{p} d F(x) \\
= & -\int_{\infty}^{0}\left(\frac{1}{2}\left(\sqrt{\left(y^{2}+4\right)}-y\right)\right)^{p} d F\left(\frac{1}{2}\left(\sqrt{\left(y^{2}+4\right)}-y\right)\right) \\
& +\int_{0}^{\infty}\left(\frac{1}{2}\left(y+\sqrt{\left(y^{2}+4\right)}\right)\right) d F\left(\frac{1}{2}\left(y+\sqrt{\left(y^{2}+4\right)}\right)\right) \\
= & \frac{1}{2} \int_{0}^{\infty}\left[\left(\frac{1}{2}\left(\sqrt{\left(y^{2}+4\right)}-y\right)\right)^{p}+\left(\frac{1}{2}\left(\sqrt{\left(y^{2}+4\right)}+y\right)\right)^{p}\right] d F(y),
\end{aligned}
$$

the last line using (5.6) and (5.7). Now let $T_{n}$ be the Chebyshev polynomial of degree $n$ defined by $T_{n}\left(x+x^{-1}\right)=x^{n}+x^{-n}$. Then, as is well known (see e.g. [11, p. 309]), $T_{0}(x) \equiv 2$ and for $n \geqslant 1$

$$
\begin{aligned}
T_{n}(x) & =\left(\frac{1}{2}\left(x+\sqrt{\left(x^{2}-4\right)}\right)\right)^{n}+\left(\frac{1}{2}\left(x-\sqrt{\left(x^{2}-4\right)}\right)\right)^{n} \\
& =x^{n}+\sum_{j=1}^{\lfloor n / 2\rfloor}(-1)^{j} \frac{n}{j}\left(\begin{array}{c}
n-j-1 \\
j-1
\end{array}\right) x^{n-2 j} .
\end{aligned}
$$

Hence the integrand of (5.8), for $p=2 k \geqslant 2$ an even integer, is equal to

$$
\begin{aligned}
i^{2 k} T_{2 k}(-i y) & =y^{2 k}+\sum_{j=1}^{k} \frac{2 k}{j}\left(\begin{array}{c}
2 k-j-1 \\
j-1
\end{array}\right) y^{2(k-j)} \\
& =\sum_{j=0}^{k}\left(\begin{array}{c}
k+j \\
k-j
\end{array}\right) \frac{2 k}{k+j} y^{2 j} .
\end{aligned}
$$

Thus from (5.8)

$$
a_{2 k}^{2 k}=\frac{1}{2} \sum_{j=0}^{k}\left(\begin{array}{l}
k+j \\
k-j
\end{array}\right) \frac{2 k}{k+j} a_{2 j}^{2 j} \quad(k \geqslant 1),
$$


from which (5.4) follows. Then, from (5.4), $a_{2 k}^{2 k} \geqslant 2 k a_{2 k-2}^{2 k-2}$, so $a_{2 k}^{2 k} \geqslant 2^{k} k$ !, from which, with Stirling's formula, we obtain (5.5).

Finally we take another look at the sequence $\left\{a_{2 k}^{2 k}\right\}=\{1,2,10,80, \ldots\}$. We note that $\left\{a_{2 k}^{2 k}\right\}$ satisfies another recurrence, 'dual' to (5.4):

$$
a_{2 k}^{2 k}=(-1)^{k-1}\left(\begin{array}{c}
2 k \\
k
\end{array}\right)+2 \sum_{j=1}^{k-1}(-1)^{k-j-1}\left(\begin{array}{c}
2 k \\
k-j
\end{array}\right) a_{2 j}^{2 j} .
$$

This can be obtained from the fact that, from $(2.32-2.33)$,

$$
d F(x)= \begin{cases}-\frac{1}{2} d F\left(x^{-1}-x\right), & 0<x<1, \\ \frac{1}{2} d F\left(x-x^{-1}\right), & x \geqslant 1,\end{cases}
$$

from which one gets easily that

$$
\int_{0}^{\infty} x^{p} d F(x)=\int_{0}^{\infty}\left|x-x^{-1}\right|^{p} d F(x) .
$$

Then (5.12) follows readily for $p=2 k$ on expanding $\left(x-x^{-1}\right)^{2 k}$, and using (2.31).

We now give a brief explanation of the connection between (5.4) and (5.12) in terms of inverse relations between pairs of sequences; see [5, p. 9].

THEOREM 7. Given a sequence $\left\{A_{n}\right\}_{n=0}^{\infty}$ and defining $B_{0}=A_{0}$ and

$$
B_{n}=\sum_{k=0}^{n} \frac{n}{n+k}\left(\begin{array}{c}
n+k \\
n-k
\end{array}\right) A_{k} \quad(n=1,2, \ldots),
$$

there is an inverse relation

$$
A_{n}=(-1)^{n}\left(\begin{array}{c}
2 n \\
n
\end{array}\right) B_{0}+\sum_{k=1}^{n} 2\left(\begin{array}{c}
2 n \\
n-k
\end{array}\right)(-1)^{n-k} B_{k} \quad(n=1,2, \ldots) .
$$

The sequence $\left\{a_{2 n}^{2 n}\right\}$ is an eigensequence of this relation (i.e. if $A_{n}=a_{2 n}^{2 n}(n=0,1, \ldots)$, then $\left.B_{n}=A_{n}(n=0,1, \ldots)\right)$.

The inverse relationship is a consequence of the binomial identity

$$
\sum_{j=k}^{n}\left(\begin{array}{c}
n+j \\
n-j
\end{array}\right) \frac{2 n}{n+j}\left(\begin{array}{c}
2 j \\
j-k
\end{array}\right)(-1)^{j-k}=\delta_{n k} \quad(0 \leqslant k \leqslant n, n \geqslant 1) .
$$

This identity can be proved by applying (5.10) to the identity

$$
(-1)^{n} T_{2 n}\left(-i\left(x-x^{-1}\right)\right)=x^{2 n}+x^{-2 n} \text {. }
$$

Theorem 7 characterizes the sequence $\{1,2,10,80,874, \ldots\}$ in a manner independent of the function $F$.

Mathematics Department

James Cook University of North Queensland

Townsville 4811, Australia

1. D. W. Boyd, "Small Salem numbers,” Duke Math. J., v. 44, 1977, pp. 315-328.

2. V. EnNola, "Conjugate algebraic integers in an interval," Proc. Amer. Math. Soc., v. 53, 1975, pp. 259-261.

3. H. Gould, Combinatorial Identities, Morgantown Printing Co., 1972.

4. I. S. Gradshteyn \& I. M. Ryzhik, Table of Integrals, Series, and Products, Academic Press, New York, 1965.

5. D. H. Greene \& D. E. Knuth, Mathematics for the Analysis of Algorithms, Birkhäuser, Boston, 1981. 
6. G. H. Hardy, J. E. Litrlewood \& G. Polya, Inequalities, 2nd ed., Cambridge Univ. Press, 1952.

7. J. HUNTER, "A generalisation of the inequality of the arithmetic-geometric means," Glasgow Math. J., v. I, 1958, pp. 149-158.

8. L. LewIn, Polylogarithms and Associated Functions, North-Holland, Amsterdam, 1981.

9. M. J. McAuley, Topics in J-Fields and a Diameter Problem, M. Sc. thesis, University of Adelaide, 1981.

10. D. S. Mitrinović, Analytic Inequalities, Springer, New York, 1970.

11. R. M. Robinson, “Intervals containing infinitely many sets of conjugate algebraic integers," Studies in Mathematical Analysis and Related Topics: Essays in Honor of George Polya. Stanford, 1962, pp. 305-315.

12. R. M. Robinson, “Algebraic equations with span less than 4," Math. Comp., v. 10, 1964, pp. 549-559

13. C. L. Siegel, "The trace of totally positive and real algebraic integers," Ann. of Math., v. 46, 1945. pp. $302-312$.

14. C. J. Sмүтн, “On the measure of totally real algebraic integers," J. Austral. Math. Soc. Ser. A, v. 30, 1980, pp. 137-149.

15. C. J. Sмyтh, “On the measure of totally real algebraic integers. II," Math. Comp., v. 37, 1981, pp. 205-208. 